\title{
Towards Detecting and Managing Information Anxiety in the ICT Industry
}

\author{
Mark Micallef \\ Department of Computer Science \\ University of Malta \\ mark.micallef@um.edu.mt
}

\author{
Chris Porter \\ Department of Computer Information Systems \\ University of Malta \\ chris.porter@um.edu.mt
}

\begin{abstract}
Information Anxiety is defined as "stress caused by the inability to access, understand, or make use of information necessary for employees to do their job". Even though it is in itself an intangible phenomenon, it is widely acknowledged and has been linked to impaired decision-making ability, information withdrawal, information avoidance, burnout and other health issues. The ICT industry is acknowledged to be a fully fledged knowledge industry. That is to say that its workers are mainly tasked with creating, understanding, applying and distributing knowledge as part of their day-to-day job. The industry is also characterised by disruptive innovations, continuously changing technologies and customers who constantly change their mind about what they want systems to do.

In this paper, we present the results of a study which tracked 18 participants for a period of one month in order to investigate the presence of information anxiety in the Maltese ICT industry. Our results indicate that information anxiety is present in nontrivial levels amongst our cohort of participants, with information overload being the predominant cause. Also, participants working in a quality assurance (QA) function are more exposed to the phenomenon as well as being exposed to a wider variety of sources of anxiety than developers. Experience is also shown to be a factor with participants being less prone to symptoms of information overload as they gain more experience in the field.
\end{abstract}

Index Terms-Information Anxiety, Software Engineering, Empirical Study

\section{INTRODUCTION}

In 1956, white-collar workers outnumbered blue-collar workers for the first time in US History [1]. Since then, a substantial portion of the commercial world (especially in western economies) has transitioned to the so-called "knowledge economy". Even though balance sheets list bank balances and material assets, it is widely acknowledged that knowledge is now the primary commodity and knowledge flows are regarded as the most important factors in the economy [2]. Knowledge workers are employees who deliver value to an organisation through the creation, storage, retrieval, dissemination and most importantly, application of knowledge. Yet, where as manual labourers tend to have very well defined and routine jobs with known inputs and specific outputs, knowledge workers have a more dynamic and less tangible context to deal with.

The ICT industry is a prime example of a knowledge industry, composed exclusively of knowledge workers. A

DOI reference number: 10.18293/SEKE2019-095 typical software engineer is expected to possess up-to-date in-depth technical knowledge as well as intimate knowledge of whatever domain she happens to be working in, so as to be able to deliver technical solutions in that domain. These technical solutions tend to be highly complex in nature and constantly evolve so as to (1) help customers remain competitive in dynamic markets; and (2) remain current with latest developments and best practices in technology. In the course of a working day, a software engineer will participate in several knowledge-centred activities. These include attending meetings in which status updates are communicated, discussing solutions to technical problems with peers, implementing technical solutions, responding to customer and management queries, fixing issues, mentoring peers, and so on. This can become overwhelming. Misra and Stokols [3] lament the rapid growth and transmission of information in the digital age and argue that this poses new challenges for individuals dealing with the onslaught of communications from multiple sources. Unless properly managed, these challenges can lead to anxiety and burnout, which in turn leads to employees loosing interest, exhibiting lower activity and feeling powerless [4]. This is where the phenomenon of information anxiety becomes more tangible.

Wurman [5] defined Information Anxiety as being the "stress caused by the inability to access, understand, or make use of information necessary for employees to do their job". Considering the contribution that the ICT industry makes to national economies [6], as well as its key role in helping other industries to grow and flourish [7], we argue that the industry needs to be aware of information anxiety, be able to detect it, and subsequently manage it.

\section{A. Research Questions}

Whilst talk of employees burning out and leaving companies can be heard around many water coolers and in the halls of conference coffee breaks, to date and to the best of our knowledge, there has been no empirical study that demonstrates the presence of information anxiety in the ICT industry. In this paper, we begin to bridge this gap by following 18 ICT professionals in Malta for a period of one month in order to investigate the following research questions: 
RQ1: To what extent is information anxiety exhibited by employees in the Maltese ICT Industry?

RQ2: What insights can be gained regarding the sources of information anxiety in the Maltese ICT industry?

\section{INFORMATION ANXIETY}

The term information anxiety was first coined by Wurman three decades ago, and was defined as "stress caused by the inability to access, understand, or make use of information necessary for employees to do their job" [5]. Since then, information anxiety has been studied in a number of contexts and from a variety of points of view. A substantial portion of the literature equates information anxiety with information overload. Hartog [8] sets out a whole set of lingo that has evolved around the concept including terms such as info-glut, techno-stress, and information addiction. Burkhardt et al. [9] define information anxiety as "a feeling of being overwhelmed that comes when confronting a large information task". Using more visual language, McCarthy [10] defines it as "a kind of stupor, a feeling that we simply can't keep up, can't read fast enough, don't know how to locate the information we need, don't have time to sort through or think about all the data surrounding us".

\section{A. Sources of Information Anxiety}

Whilst the tendency in the literature is to equate information anxiety with information overload, we argue that knowledge needs in the ICT are so complex that it is worth considering Wurman's [5] wider definition and his explanation about the causes of the phenomenon. Wurman states that information anxiety can be caused by one or more of five possible causes: (1) an individual's inability to understand the information required to carry out a task; (2) feeling overwhelmed by the amount of information that needs to be understood in order to complete a task (information overload); (3) someone not knowing whether the information required to complete a task exists or not; (4) knowing that information exists but not knowing where to find it; and finally (5) knowing where the information is but not having access to it.

\section{B. Effects of Information Anxiety}

Several studies have been carried out with the intent of uncovering the effect of information anxiety on employees. The three most common themes to emerge are (1) an impact on job performance; (2) psychological impact; and (3) a detrimental effect on the health of individuals.

1) Impact on Job Performance: After studying managers in a cross-national survey, Waddington [11] reports that $43 \%$ of managers claimed that information anxiety impaired their decision-making ability. A decade later, Williams [12] reported similar findings on impaired judgement in a similar study. Bawden and Robinson [13] claim that damage-limitation strategies resorted to by employees who were exposed to information anxiety tend to reduce their effectiveness and result in a negative impact on their employing organisation. Misra and Stokols [3] studied 484 undergraduate students and found that those exposed to high levels of information anxiety devoted less time to contemplative activities. In the context of a knowledge organisation, this would result in employees not investing the time in innovation that is essential for maintaining a competitive edge.

2) Psychological Impact: Katopol reports that individuals exposed to information anxiety can have any combination of sensations including feeling overwhelmed, intimidated, fearful, lost, threatened, stressed, uncomfortable and/or timid [14]. Fox reports that the phenomenon leaves employees feeling a sense of loss of control [15] whilst Waddington [11] found a link to tension and reduced job satisfaction.

\section{Detrimental effect on Health}

In more severe and prolonged cases, information anxiety has been observed to affect individual's physical health. Misra and Stokols [3] positively correlated higher levels of perceived information anxiety with incidences of physical health problems. Waddington [11] also reports that $42 \%$ of managers in his study believe that information overload affects their physical health. Similar conclusions were reached by Ifijeh [16], as well as Bawden and Robinson [13].

\section{Measuring Information Anxiety}

The main stumbling block with addressing information anxiety is the fact that it is difficult to detect before it reaches harmful levels. A review of the literature revealed two alternatives for measuring the levels of information anxiety in individuals.

Based on the argument in health research communities that the impact of objectively stressful events depends largely on one's perception of their stressfulness [17], Cohen et al. [18] propose their Perceived Stress Scale (PSS). The method involves asking participants to answer 14 questions with one of never, almost never, sometimes, fairly often or very often. The answers are assigned numerical values of 0 through 4 for questions with a negative bias $^{1}$ and 4 through 0 for questions with a positive bias ${ }^{2}$. The PSS score is calculated by summing the scores of all 14 questions and through three case studies, Cohen et al. showed the measure to be a reliable and sound predictor of various psychological indicators such as depressive symptomatology and social anxiety.

In order to assess information anxiety in the Canadian Air Force, Girard and Allison [19] used Wurman's [5] five causes of information anxiety as a starting point. Participants in their three studies rated their agreement with statements on a scale of 1 (strongly disagree) to 5 (strongly agree). The statements were as follows:

1) I would not understand the data required to complete this task.

2) I would feel overwhelmed by the amount of data to be understood to complete this task.

\footnotetext{
${ }^{1}$ Example: In the last month, how often have you felt nervous or stressed?

${ }^{2}$ Example: In the last month, how often have you felt the you were on top of things?
} 


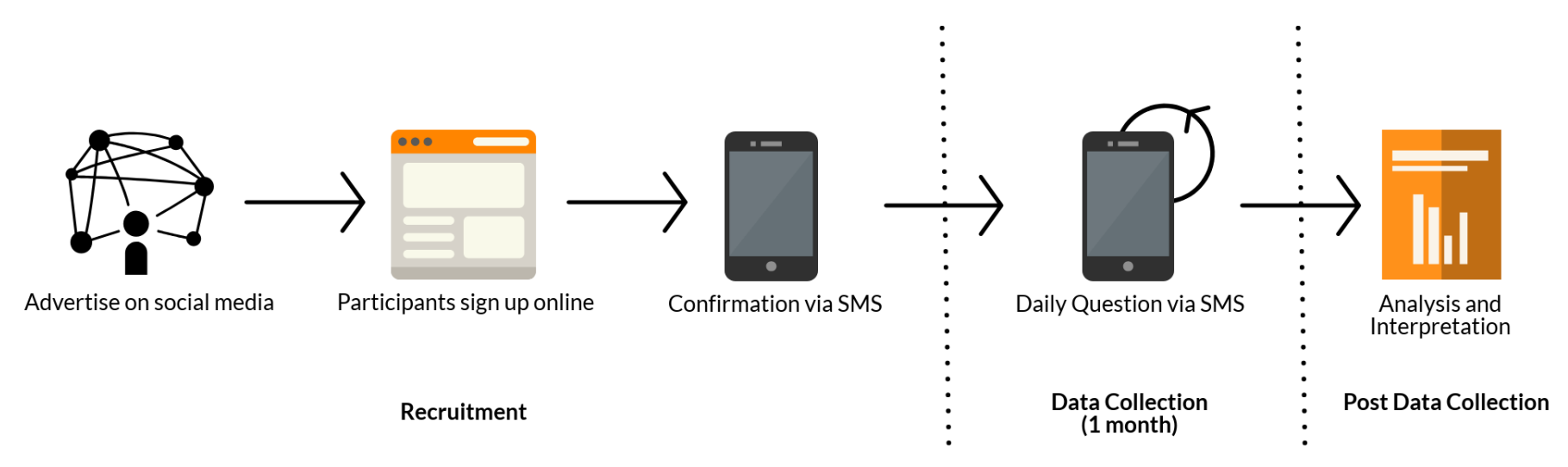

Fig. 1. An overview of the research protocol adopted for this study.

3) I would not know if certain data necessary for this task exists.

4) I would not know where to find data necessary for this task.

5) I would know exactly where to find the data, but I would not have the key to access it.

By summing up the ratings of individual components, Girard and Allison were able to measure the overall level of information anxiety experienced by an individual at a particular point in time. Although simple in nature, the measure is expressive both in terms of overall information anxiety, as well as in terms of the contribution of individual sources of anxiety.

\section{Methodology}

The research protocol was designed with the goal of regularly collecting empirical data from participants over a period of one month whilst minimising disruption to participants' daily routines. When considering the two alternatives for measuring information anxiety discussed in Section II-D, Cohen et al.'s approach [18] was ruled out because requiring participants to answer 14 questions on a regular basis would be disruptive. Instead, a modified version of Girard and Allison's [19] survey instrument was adopted. The method was aligned to the requirements of this study such that only one of the questions was asked to each participant every day. This reduced the disruption caused to participants whilst also collecting multiple data points as we followed individuals over a period of one month.

A survey mechanism utilising SMS technology was designed and implemented. As depicted in Figure 1, the study was partitioned into three phases. In the first phase, participants were recruited by means of adverts in social media groups that directed interested people to a sign-up form that collected some basic demographic information. They were later asked to confirm their participation via SMS so as to ensure that we were able to communicate with them over that medium.

The second phase consisted of data collection. Every working day for a month, participants received one question via

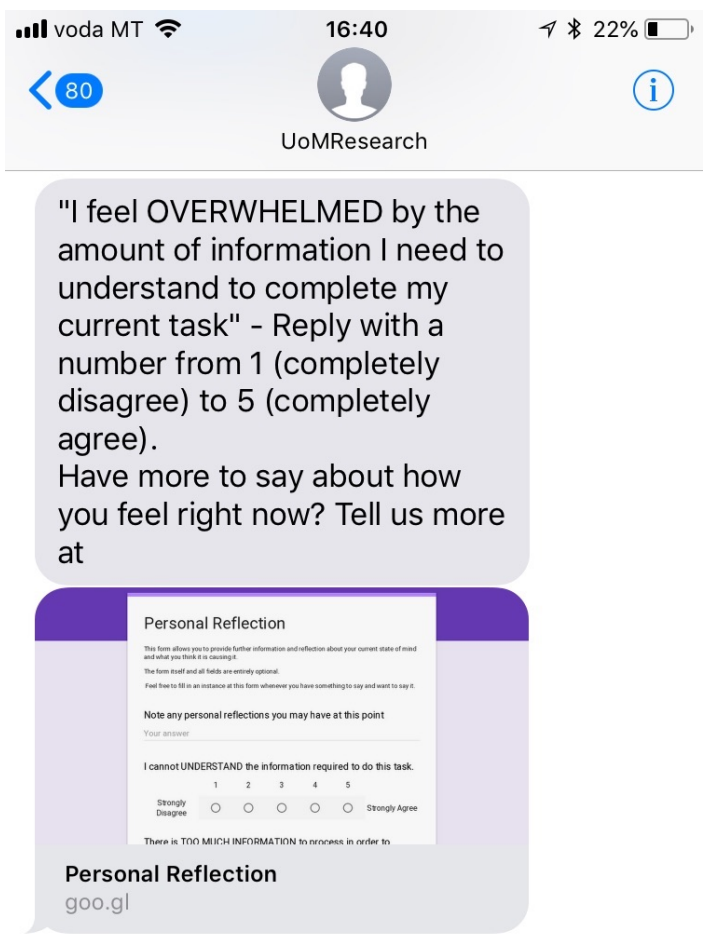

Fig. 2. A typical question sent to participants via SMS, including a link to the personal reflection form.

text message similar to the one shown in Figure 2 every day at $11 \mathrm{am}$. The choice of question was randomised but once a question was asked, it would not be asked again before all other questions had been asked to the same participant. The time that questions were sent to participants was chosen deliberately so as to maximise the chance that participants have arrived at work, gone through their morning rituals and have started working. In order to minimise disruption, candidates were required to reply to each question by a text message containing a number between 1 (strongly disagree) and 5 (strongly agree). Participants were also provided with a link to a personal reflection form whereby they could optionally 
provide further insight.

Finally, after the data collection period was over, the data was collated, analysed and interpreted.

\section{Results}

In this section, we present a digest of the key observations we gained from analysing the data collected during the study.

\section{A. Participant Demographics}

Eighteen participants signed up and participated in the study. Four demographic attributes were collected as information about participants: role, industry, experience and level of education. There was a relatively uniform distribution in all attributes except for education in which $11 \%$ of participants were educated to post-secondary level, $78 \%$ to undergraduate level and $11 \%$ to postgraduate levels. Participants were highly responsive during the study when it came to SMS responses, with a mean response rate of $79 \%$. However, no participants availed themselves of the online reflection form to provide more unstructured information.

\section{B. Initial Analysis}

An initial analysis of the data revealed that when one analyses the cohort as a whole, only $17 \%$ of responses indicated levels of information anxiety. This was interesting to us as it went contrary to our initial hypothesis that information anxiety is significant problem in the industry. However, as we dissected the data further to consider participant demographics and individual sources of information anxiety, a more comprehensive picture emerged.

When one considers individual sources of information anxiety, it turns out that eight participants $(44 \%)$ had a mean rating higher than three for at least one source. All eight of these participants indicated that information overload was the main cause of anxiety, with five participants indicating at least one more cause.

\section{The influence of job role}

Figure 3 plots a box chart showing the distribution of ratings provided by participants for each source of information anxiety, as well as the combined ratings of all sources, split by job role. One can immediately notice that job role seems to have a determining influence on the overall levels of anxiety registered by individuals. When considering all five sources of anxiety collectively, QA analysts registered comparatively high levels of anxiety with a median value of 2.5 and an upper quartile rating of 4 . This contrasts with an upper quartile rating of 2 and 3 for software engineers and project leaders respectively.

Software engineers exhibited arguably neglible ratings across all sources of information anxiety except for information overload. The median of 2.5 and upper quartile value of 4 here indicates that there is a significant perception amongst software engineers in our cohort that they have too much information to process and feel overwhelmed by it. Furthermore, both QA analysts and project leaders exhibit higher levels of anxiety as a result of information overload with over $50 \%$ of ratings from QA analysts being at or above 3.

Consistent with the other two roles, the QA Analyst cohort exhibited was mostly affected by information overload. However, the same cohort also has significant complaints of participants struggling with understanding, being unable to locate information and not having access to information. Project leaders have exposure to a wider range of anxiety sources than developers but the levels of complaints for the upper quartile only ever go beyond 3 for information overload.

\section{The influence of experience}

The most inexperienced participants reported the least problems related to being unable to understand information related to do their job. The highest problem in this area surfaced amongst participants with 6-10 years of experience in the industry. Conversely, participants with 3-5 years of experience reported the highest levels of information anxiety with $39 \%$ of information overload ratings for this cohort being 4 or 5 . The data indicates that as participants gain experienced, they suffer less from information overload.

\section{E. The influence of industry}

We also analysed data from the point of view of industries in which participants worked. However, except for a marginally higher level of anxiety being reported by participants in the Gaming industry, the data indicates that the domain in which participants work does not have an obvious influence on the levels of information anxiety experienced.

\section{F. Threats to Validity}

The study suffers from two main threats to validity. Firstly, the sample size of 18 is not necessarily representative of the industry as a whole. Secondly, the duration of the study (1 month) when compared to the lifetime of a typical project. Having said that, the study is, to the best of our knowledge, the first of its kind to shed light on the levels of information anxiety in the ICT industry and lessons learned here will be taken forward to larger and more longitutional studies that are being planned by the authors.

\section{DISCUSSION}

In this section we discuss the results of the exercise in the context of the research questions specified in Section I-A.

\section{A. Presence of Information Anxiety in the ICT Industry}

Although the data does not indicate an outright epidemic when it comes to information anxiety amongst our participants, it does indicate that there is cause for concern. This can be better appreciated when one considers that results obtained using Girrard and Allison's [19] formula for calculating information anxiety can be misleading if one does not consider that it is an aggregating formula. This is because, our data shows that an individual is most likely to be exposed to one source of information anxiety. There were cases were individuals were exposed to two or at most three sources at any point 


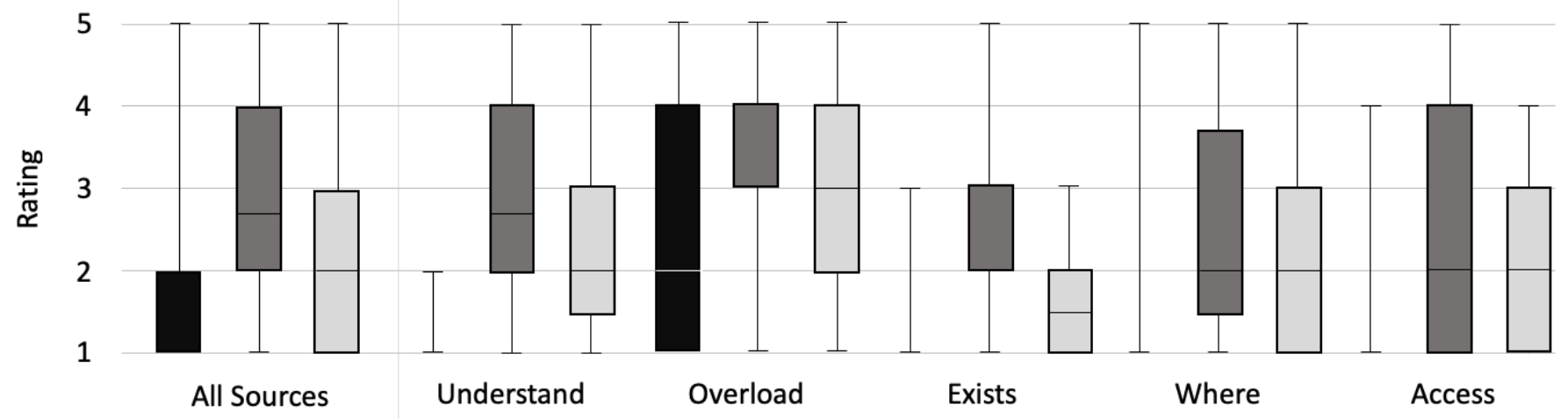

Fig. 3. Distribution of ratings for sources of information anxiety for Software Engineers (black), QA Analysts (dark grey) and Project Leaders (light gray).

in time but these were very rare. It is therefore likely that Girrard and Allison's formula will even out to a mean, as evidenced by their own studies where the mean value for anxiety readings hovered around 2.5 . However, this does not mean that no information anxiety is present. Rather, we argue that a prolonged high rating for even one source of anxiety on one participant should be cause for concern and trigger an investigation to understand the circumstances leading to this.

Whilst it was out of scope for this particular study, individual employees ratings should also be examined over time to look for patterns that might be detrimental to employees psychological health. For example, regular short bursts of high anxiety would produce low average scores but employees could still have significantly negative experiences as a result.

Notwithstanding the small sample of participants, in answer to RQ1, the data collected for this study suggests that information anxiety is sufficiently present in the Maltese ICT industry to cause employers to devise ways of detecting it and measuring it.

\section{B. Insights regarding the sources of Information Anxiety}

The results from this study lead us to make three conclusions when it comes to the sources of information anxiety.

1) The predominance of Information Overload: Information overload was consistently shown to be the predominant cause of anxiety amongst our participants when compare to the other four sources regardless of job role, experience level or industry. This indicates that the literature's focus on information overload is well founded but not complete. For example, QA analysts demonstrated elevated levels of anxiety across multiple sources of anxiety. In such cases, whilst employees are still being suffering from anxiety the required solutions would be different to those required when the root cause is information overload.

2) QA Analysts have an anxiety-inducing job: The indication that QA Analysts suffer from higher levels of anxiety than other job roles is further explained when one examines the individual sub-components of information anxiety as experienced by people in this role. QA analysts tend to act as bridge communicators between the technical side of an organisation (software engineers) and the so called product side of the business. The need to straddle two different worlds in which people often speak different languages and dialects. That is to say that whilst a software engineer might speak in terms of concepts such as algorithms, efficiency, load balancing and so on, a product-side employee would speak in terms of features, customers and other domain-specific concepts. QA analysts are responsible for ensuring that the technical side of the business delivers the right artefacts to the product side of the business and it is this straddling of two worlds that likely leads to elevated levels of anxiety.

3) Experience is a factor: One of the finding of the study is that symptoms of information overload decrease amongst participants with more experience. This is probably due to experienced individuals being able to develop soft skills for setting up barriers against information overload. For example, the simple act of saying No, I cannot deliver this extra feature in the next 2 weeks. has the effect of shielding an individual against any anxiety that might have resulted from committing to the extra task and setting himself up for failure. Individuals who have been around for longer are more capable of gauging the effect that a task might have of them and are more comfortable refuting that work.

\section{RELATED WORK}

As discussed in Section I, to date and to the best of our knowledge, there has been no empirical study that demonstrates the presence of information anxiety in the ICT industry. The work the came closest to this is that of work that Mäntylä et al. [4], who did not explicitly discuss information anxiety but did discuss the ICT sector and highlight a number of related concepts and symptoms.

Mäntylä et al. claim that employees in the ICT sector are susceptible to "psychological diseases such as burnout, which lead developers to lose interest, exhibit lower activity and/or feel powerless" [4]. They propose a set of metrics called the $V A D$ metrics, named after the first letter of three attributes which they measure: (V)alence, the level of enjoyment exhibited by individuals carrying out a task; (A)rousal, the level of alertness and readiness to act; and (D)ominance, the level to 
which individuals are in control of their task. Using a database of 13,915 manually rated English words as created by Warriner [20], Mäntylä et al. calculated VAD scores on 700,000 $\mathrm{Jira}^{3}$ tickets containing over 2,000,000 comments. This resulted in a number of interesting conclusions regarding how valence, arousal and dominance in individuals varies based on how long a ticket remains open, how its priority changes and when it is eventually resolved. For example, their results indicate that burnout (low valence, low dominance and high arousal) is more likely when working on high priority issues that take a long time to resolve. Whilst Mäntylä et al. do not make the link with information anxiety, as discussed in Section I, software engineering is a knowledge-intensive activity and a prolonged unsolved issue is likely to be caused by a lack of understanding, information overload or issues with finding and/or accessing relevant information.

\section{CONCLUSION AND FUtURE WORK}

In this paper we discussed the methodology and results of a study that was designed and executed to shed light on the presence of information anxiety in the ICT industry. By tracking anxiety levels amongst 18 participants on a daily basis for one month, we discovered that information anxiety is indeed present at non-trivial levels with information overload being the predominant cause. We also discovered that QA Analysts are more likely to suffer from the phenomenon, as are less experienced individuals. Finally, we discovered that the domain in which employees work does not have a significant bearing on anxiety levels.

\section{A. Recommendations}

This study contributes a non-intrusive data collection protocol that can help companies shed light on levels of information anxiety amongst their employees. Implementing a similar mechanism which collects data at possibly less regular intervals using company's intranet or time-sheet system would provide managers with valuable empirical data. This data can subsequently be analysed and tracked such that any emerging problems with information anxiety can be investigated and addressed before they cause substantial harm. Furthermore, the knowledge that employees in certain job roles are more susceptible to the phenomenon should lead to a review how these roles could be restructured in order to lessen the likelihood of information anxiety emerging.

\section{B. Future Work}

Having taken the first step towards investigating information anxiety in the ICT industry, we plan continuing this work across two prongs of research. number of related projects in the near future. Firstly, we would like to set up a longitudinal study which spans a longer period, ideally a year or more. This would allow us to gain insights into how and why anxiety levels change over time. We anticipate that the main

\footnotetext{
${ }^{3}$ Jira is a commonly used tool in the ICT industry which enables stakeholders to report issues with software (or request new features) and track work and discussions by developers whilst development progresses.
}

problem with such a study would be maintaining participant engagement, and to this end we are currently investigating the possibility of utilising gamification to achieve this.

Secondly, we would like to investigate the sources of anxiety in more detail. In particular, we are interested in understanding if there are any relationships between specific components and whether these are amplified in particular contexts. Insights gained in this area could lead future studies with a more refined focus on specific contexts or sources of anxiety.

\section{REFERENCES}

[1] J. Kenway, E. Bullen, J. Fahey, and S. Robb, Haunting the knowledge economy. Routledge, 2006, vol. 6.

[2] L. Chinho and T. Shu-Mei, "The implementation gaps for the knowledge management system," Industrial Management \& Data Systems, vol. 105, no. 2, pp. 208-222, February 2005. [Online]. Available: http://dx.doi.org/10.1108/02635570510583334

[3] S. Misra and D. Stokols, "Psychological and health outcomes of perceived information overload," Environment and behavior, vol. 44, no. 6 , pp. 737-759, 2012.

[4] M. Mäntylä, B. Adams, G. Destefanis, D. Graziotin, and M. Ortu, "Mining valence, arousal, and dominance: possibilities for detecting burnout and productivity?" in Proceedings of the 13th International Conference on Mining Software Repositories. ACM, 2016, pp. 247258.

[5] R. S. Wurman, Information Anxiety. Doubleday, 1989. [Online]. Available: http://books.google.com/books?id=f8ZoAAAAIAAJ

[6] M. K. Sein and G. Harindranath, "Conceptualizing the ict artifact: Toward understanding the role of ict in national development," The Information Society, vol. 20, no. 1, pp. 15-24, 2004.

[7] D. Pilat and A. Wölfl, "Ict production and ict use: What role in aggregate productivity growth?" The Economic impact of ICT-measurement, evidence, and implications, pp. 85-104, 2004.

[8] P. Hartog, "A generation of information anxiety: Refinements and recommendations," The Christian Librarian, vol. 60, no. 1, p. 8, 2017.

[9] J. M. Burkhardt, M. C. MacDonald, and A. J. Rathemacher, Teaching information literacy: 50 standards-based exercises for college students. American Library Association, 2010.

[10] M. McCarthy, "Mastering the information age," Los Angeles: Jeremy P. Tarcher, 1991.

[11] P. Waddington, "Dying for information? a report on the effects of information overload in the uk and worldwide," British library research and innovation report, pp. 49-52, 1997.

[12] C. J. Williams, Reassessing the role of anxiety in information seeking. University of North Texas, 2008.

[13] D. Bawden and L. Robinson, "The dark side of information: overload, anxiety and other paradoxes and pathologies," Journal of information science, vol. 35 , no. 2, pp. 180-191, 2009.

[14] P. F. Katopol, "Information anxiety and african-american students in a graduate education program." Education Libraries, vol. 35, pp. 5-14, 2012.

[15] J. Fox, "Conquering information anxiety. relief from your data glut starts here," 1998. [Online]. Available: http://www.ibtpep.com/articles/dataglutrelief.doc

[16] G. Ifijeh, "Information explosion and university libraries: Current trends and strategies for intervention," Chinese Librarianship: an International Electronic Journal, 2010.

[17] R. S. Lazarus, "Psychological stress and coping in adaptation and illness," The International journal of psychiatry in medicine, vol. 5, no. 4, pp. 321-333, 1974.

[18] S. Cohen, T. Kamarck, and R. Mermelstein, "A global measure of perceived stress," Journal of health and social behavior, pp. 385-396, 1983.

[19] J. Girard and M. Allison, "Information anxiety: Fact, fable or fallacy," Electronic Journal of Knowledge Management, vol. 6, no. 2, pp. 111124, 2008.

[20] A. B. Warriner, V. Kuperman, and M. Brysbaert, "Norms of valence, arousal, and dominance for 13,915 english lemmas," Behavior research methods, vol. 45, no. 4, pp. 1191-1207, 2013. 